\title{
Presubiculum Stimulation In Vivo Evokes Distinct Oscillations in Superficial and Deep Entorhinal Cortex Layers in Chronic Epileptic Rats
}

\author{
Else A. Tolner, ${ }^{1,5}$ Fabian Kloosterman, ${ }^{2}$ Erwin A. van Vliet, ${ }^{1,3}$ Menno P. Witter, ${ }^{4}$ Fernando H. Lopes da Silva, ${ }^{1}$ and \\ Jan A. Gorter ${ }^{1,3}$ \\ ${ }^{1}$ Swammerdam Institute of Life Sciences, Center for Neuroscience, University of Amsterdam, Graduate School of Neurosciences Amsterdam, 1098 SM \\ Amsterdam, The Netherlands, ${ }^{2}$ Picower Center for Learning and Memory, Massachusetts Institute of Technology, Cambridge, Massachusetts 02139, \\ ${ }^{3}$ Stichting Epilepsie Instellingen Nederland, 2103 SW Heemstede, The Netherlands, ${ }^{4}$ Department of Anatomy, Vrije Universiteit Medical Center, Research \\ Institute of Neurosciences, Graduate School of Neurosciences Amsterdam, 1081 BT Amsterdam, The Netherlands, and 5Institute of Neurophysiology, \\ Charité University Medicine Berlin, 10117 Berlin, Germany
}

The characteristic cell loss in layer III of the medial entorhinal area (MEA-III) in human mesial temporal lobe epilepsy is reproduced in the rat kainate model of the disease. To understand how this cell loss affects the functional properties of the MEA, we investigated whether projections from the presubiculum (prS), providing a main input to the MEA-III, are altered in this epileptic rat model. Injections of an anterograde tracer in the prS revealed bilateral projection fibers mainly to the MEA-III in both control and chronic epileptic rats. We further examined the prS-MEA circuitry using a 16-channel electrode probe covering the MEA in anesthetized control and chronic epileptic rats. With a second 16-channel probe, we recorded signals in the hippocampus. Current source density analysis indicated that, after prS double-pulse stimulation, afterdischarges in the form of oscillations $(20-45 \mathrm{~Hz})$ occurred that were confined to the superficial layers of the MEA in all epileptic rats displaying MEA-III neuronal loss. Slower oscillations (theta range) were occasionally observed in the deep MEA layers and the dentate gyrus. This kind of oscillation was never observed in control rats. We conclude that dynamical changes occur in an extensive network within the temporal lobe in epileptic rats, manifested as different kinds of oscillations, the characteristics of which depend on local properties of particular subareas. These findings emphasize the significance of the entorhinal cortex in temporal lobe epilepsy and suggest that the superficial cell layers could play an important role in distributing oscillatory activity.

Key words: reorganization; epileptogenesis; parahippocampal region; current source density; field potentials; kainate

\section{Introduction}

The entorhinal cortex (EC) and hippocampus form an important functional system involved in memory formation and stress adaptation (Witter et al., 2000b). Pathological changes in this system are apparent in mesial temporal lobe epilepsy (MTLE), but the exact role of both regions in the process of epileptogenesis is not clear (Heinemann et al., 1993; Jones, 1993; Lothman et al., 1996; Scharfman, 2002). In patients with MTLE, seizures often commence in the EC, suggesting an involvement of the EC in

\footnotetext{
Received March 24, 2005; revised June 9, 2005; accepted Aug. 12, 2005.

This work was supported by The Netherlands Organization for Scientific Research Grant 90347078 (E.A.T., J.A.G.) and the National Epilepsy Foundation "Power of the Small" Grant 20-03 (J.A.G.). We thank Theo van Haeften (Department of Anatomy, Vrije Universiteit Medical Center, Amsterdam, The Netherlands; currently, Department of Biochemistry, Cell Biology and Histology, Faculty of Veterinary Medicine, University of Utrecht, Utrecht, The Netherlands) for assisting with anatomical tracings, Wijnand Takkenberg and Erik Manders (Center for Advanced Microscopy, Swammerdam Institute for Life Sciences, Amsterdam, The Netherlands) for assisting with confocal imaging, Stiliyan N. Kalitzin (Stichting Epilepsie Instellingen Nederland, Heemstede, The Netherlands) for help on the wavelet analysis, and Prof. Uwe Heinemann and Prof. Wytse Wadman for helpful discussions and critical reading of this manuscript.

Correspondence should be addressed to Dr. Jan A. Gorter, Kruislaan 320, 1098 SM Amsterdam, The Netherlands. E-mail: gorter@science.uva.nl.

DOI:10.1523/JNEUROSCI.1165-05.2005

Copyright $\odot 2005$ Society for Neuroscience $\quad$ 0270-6474/05/258755-11\$15.00/0
}

epileptogenesis (Spencer and Spencer, 1994; Bartolomei et al., 2004). In support of this, abnormal neuronal activity has been recorded in the EC of epileptic patients (Colder et al., 1996; Bragin et al., 1999). Several experiments in animal models of epilepsy, both in vivo and in vitro, have further strengthened the idea that the EC is functionally involved in the development and possible maintenance of epileptiform activity in the temporal lobe (Walther et al., 1986; Pare et al., 1992; Heinemann et al., 1993; Bear et al., 1996; Fountain et al., 1998; Gloveli et al., 1998; Scharfman et al., 1998; Bragin et al., 2002; Kobayashi et al., 2003). Based on studies in hippocampal-entorhinal slices under conditions of enhanced excitability, it has been hypothesized that, in epilepsy, transmission of synchronous activity from deep to superficial layers is facilitated (Jones and Lambert, 1990; Iijima et al., 1996; Dickson and Alonso, 1997; Lopantsev and Avoli, 1998; Scharfman, 2002). In a previous study in chronic epileptic rats (kainate model), we observed that stimulation of the subiculum, which preferentially projects to deep EC layers (Kohler, 1985; van Haeften et al., 1995; Kloosterman et al., 2003a), evokes oscillatory responses in superficial entorhinal layers (Tolner et al., 2005).

In patients with MTLE, the superficial part of the rostral EC presents marked cell loss that is particularly noticeable in layer III 
(Du et al., 1993). This characteristic cell loss in layer III of the EC has been reproduced in several rat models of MTLE after induction of a status epilepticus (SE) (Du et al., 1995; van Vliet et al., 2004) and was found to be mostly confined to the medial area of the entorhinal cortex (MEA). In view of the strong degeneration of the MEA-III, we asked how the functional properties of the MEA and associated hippocampal system would be affected in the rat MTLE model.

A main direct anatomical input to the MEA-III in rats arises from the superficial layers of the presubiculum (prS) (Kohler, 1984, 1985; Caballero-Bleda and Witter, 1993; Eid et al., 1996; van Haeften et al., 1997; Honda and Ishizuka, 2004), an area that appears mostly unaffected in chronic epilepsy in both humans (Mathern et al., 1996) and rats (Pitkanen et al., 1995; Tolner et al., 2001; van Vliet et al., 2004). To understand better the changes that occur in the MEA in chronic epilepsy, we studied, first, the anatomy of the prS-MEA connection in chronic epileptic rats and, second, the physiology of the prS-MEA circuitry in vivo by evoked field potential recordings and current source density (CSD) analysis.

\section{Materials and Methods}

Animals. Twenty-five adult male Sprague Dawley rats (Harlan CPB, Zeist, The Netherlands) of 250-550 g were used in this study. The rats were housed in individual cages under a controlled environment $(21 \pm$ $1{ }^{\circ} \mathrm{C}$; humidity, $60 \%$; lights on from 8:00 A.M. to 8:00 P.M.). Food and water were available ad libitum. For the induction of a SE, 13 rats received intraperitoneal kainic acid (KA) injections; 12 control animals received intraperitoneal saline injections. Rats were given hourly injections of KA (5 mg/kg, i.p.; Opika-1 kainic acid; Ocean Produce International, Canada, Shelburne, Nova Scotia, Canada) or saline, and seizure activity was rated behaviorally according to Racine's scale [class I-V seizures (Racine, 1972)] during the injection period and at least $4 \mathrm{~h}$ thereafter (Tolner et al., 2003). KA injections were continued until class IV and V seizures were elicited for $\geq 3 \mathrm{~h}$, which constitutes the SE. Rats were given sugar water for a week after treatment. The KA-induced SE rats will be referred to as KA rats. In all but one of the KA rats, we observed spontaneous seizures, starting from $\sim 2$ weeks after SE induction. The experimental protocols followed the European Communities Council Directive 86/ 609/EEC and the Dutch Experiments on Animal Act (1997) and were approved by the Animal Welfare Committee of the University.

Surgery for anatomical tracing. Tracing experiments were performed in seven KA rats and four saline controls at 2-6 months after KA-induced SE. Rats were anesthetized with an intramuscular injection of ketamine (57 mg/kg; Alfasan, Woerden, The Netherlands) and xylazine $(9 \mathrm{mg} / \mathrm{kg}$; Bayer AG, Leverkusen, Germany) and mounted in a stereotaxic frame. Additional doses were administered if the withdrawal reflex after hindpaw pinching returned. A glass micropipette (GC150F-15; Clark, Reading, UK) with a tip diameter of $10-15 \mu \mathrm{m}$ was filled with a $5 \%$ solution of biotinylated dextran amine, molecular weight of 10,000 (BDA) (Invitrogen, Eugene, OR) in $0.01 \mathrm{~m} \mathrm{PBS,} \mathrm{pH} \mathrm{7.4.} \mathrm{Small} \mathrm{holes} \mathrm{were} \mathrm{made} \mathrm{in} \mathrm{the}$ skull of the rat, and the pipette was lowered into the desired area at the following coordinates: anteroposterior (AP), between 7.0 and $7.9 \mathrm{~mm}$ posterior to bregma; mediolateral $(\mathrm{ML}), 3.5-4.1 \mathrm{~mm}$; dorsoventral (DV), 3.8-4.0 mm below cortical surface. Injection coordinates were derived from an anatomical map that described the topographical arrangement of presubicular projections to the EC (Caballero-Bleda and Witter, 1993) and from a stereotaxic atlas of the rat brain (Paxinos and Watson, 1998). Coordinates were selected such that injections were targeted to the superficial layers of the prS at a middle to middorsal level along the longitudinal axis of the prS. From these levels, the prS projections in control rats have been shown to terminate in the MEA at a middle to midventral level along the longitudinal axis. BDA was injected by applying a small positive-pulsed DC onto the micropipette ( $6 \mu \mathrm{A} ; 7 \mathrm{~s}$ on, $7 \mathrm{~s}$ off) for $20 \mathrm{~min}$. After 9-13 d survival, the animals were deeply anesthetized with sodium pentobarbital (Nembutal, i.p.; $60 \mathrm{mg} / \mathrm{kg}$ body weight; Sanofi Santé, Maassluis, The Netherlands) before transcardial perfusion (see below).

Surgery for field recordings. Field potential recordings were performed in six KA rats and eight saline controls at 2-4 months after KA-induced SE. Rats were anesthetized with an intramuscular injection of ketamine and xylazine as described for anatomical tracing. Surgical procedures and electrode placement, optimized to get reliable evoked field potentials, were similar to those described previously (Kloosterman et al., 2003b). Briefly, a 16-channel silicon probe (100 $\mu \mathrm{m}$ interelectrode spacing; kindly provided by the University of Michigan Center for Neural Communication Technology sponsored by National Institutes of Health-National Center for Research Resources Grant P41-RR09754) was lowered into the dorsal part of the MEA at a location $1.0-1.5 \mathrm{~mm}$ posterior to bregma, $4.7-5.0 \mathrm{~mm}$ lateral, angled at $55^{\circ}$ in the sagittal plane. With this approach, the probe penetrated all layers approximately perpendicularly (see Fig. $2 B$ ). The length of the probe restricted us to record in the dorsal MEA, instead of the ventral MEA. Inserted at the angle needed to cross the MEA layers perpendicularly, it was not feasible to reach more ventral levels of the MEA. As shown in Figure $1 G-I$, the MEA-III is degenerated also at the more dorsal levels of the MEA in chronic KA rats. In three KA and six control rats, a second 16-channel silicon probe was positioned into the hippocampal formation, such that it covered both area CA1 and the superior blade of the dentate gyrus (DG) (see Fig. 7D). It has been shown that projections from the prS to EC are topographically organized in such a way that the dorsoventral position of the origin in the prS determines the dorsoventral level of termination in the EC (CaballeroBleda and Witter, 1993). With the EC recording in the dorsal MEA, the stimulation site needs to be in a dorsal part of the prS in the proximity of the coordinates used during the tracing experiments. Bipolar stimulation electrodes (70 $\mu \mathrm{m}$ insulated stainless steel; $300-400 \mu \mathrm{m}$ vertical tip separation) were positioned in the dorsal prS (AP, between 7.0 and $7.9 \mathrm{~mm}$ posterior to bregma; ML, 2.8-2.9 mm; DV, 2.7-3.0 mm below cortical surface) (see Fig. $2 A$ ). The stimulated area included both superficial and deep layers of the prS. A standard paired-pulse protocol (pulse duration, $0.1 \mathrm{~ms}$ positive, $0.1 \mathrm{~ms}$ negative; current, $0.075-0.999 \mathrm{~mA}$ ) with a $100 \mathrm{~ms}$ interpulse interval and a typical repetition rate of $0.08 \mathrm{~Hz}$ was used.

Recording of field activity. Local field potentials were recorded relative to a silver chloride wire in the frontal cortex. Wideband signals were amplified $200 \times$ by custom-made amplifiers and digitized through a CED $1401+$ system equipped with a 32-channel simultaneous sample-andhold extension board and Signal software (Cambridge Electronic Design, Cambridge, UK; sampling at $10 \mathrm{kHz}$ ). At the end of an experiment, the locations of the stimulation and recording sites were marked by an electrolytic lesion (stainless-steel electrodes: two $400 \mathrm{~ms}$ positive current pulses of $400 \mu \mathrm{A}$; silicon probe: injection of two $15-20 \mu \mathrm{A}$ positive current pulses into the two outer channels for $10 \mathrm{~s}$ ).

Histology and cell counts. After completion of an experiment, rats were perfused through the ascending aorta with $300 \mathrm{ml}$ of $0.37 \% \mathrm{Na}_{2} \mathrm{~S}$ solution and $300 \mathrm{ml}$ of $4 \%$ paraformaldehyde with $0.2 \%$ glutaraldehyde in 0.1 M phosphate buffer, $\mathrm{pH}$ 7.4. Brains were postfixed overnight at $4^{\circ} \mathrm{C}$ and cryoprotected in $30 \%$ phosphate-buffered sucrose solution, $\mathrm{pH}$ 7.4. After an overnight incubation at $4^{\circ} \mathrm{C}$, brain parts were frozen in isopentane $\left(-25^{\circ} \mathrm{C}\right)$ and stored at $-80^{\circ} \mathrm{C}$ until sectioning.

For analysis of the anatomical tracings, horizontal sections $(40 \mu \mathrm{m}$ thick) were cut on a sliding microtome and collected in PBS $(0.05 \mathrm{M} ; \mathrm{pH}$ 7.4). Sections from different dorsoventral levels of the brain were selected to be stained for the presence of BDA. All washes and incubations were performed at room temperature. Sections were washed once in $0.05 \mathrm{M}$ Tris buffer, $\mathrm{pH} 7.6$, and incubated for $30 \mathrm{~min}$ in $0.3 \% \mathrm{H}_{2} \mathrm{O}_{2}$ in Tris buffer to inactivate endogenous peroxidase. After two successive washes in Tris buffer, sections were incubated for $1.5 \mathrm{~h}$ in an avidin-biotin peroxidase complex (Vectastain; Vector Laboratories, Burlingame, CA; prepared according to the manufacturer's recommendations) in Tris buffer containing 0.5\% Triton X-100 (Tris-Tx). After several rinses in Tris-Tx, the labeling was visualized by incubation in 3,3' -diaminobenzidine tetrahydrochloride (DAB) medium: 3 mg of DAB (Sigma, Deisenhofen, Germany) and $75 \mu \mathrm{l}$ of $1 \% \mathrm{H}_{2} \mathrm{O}_{2}$ in a $10 \mathrm{ml}$ solution of Tris-Tx. During this reaction, sections were inspected at intervals with a microscope. When maximal specific staining with minimal background was reached, the 

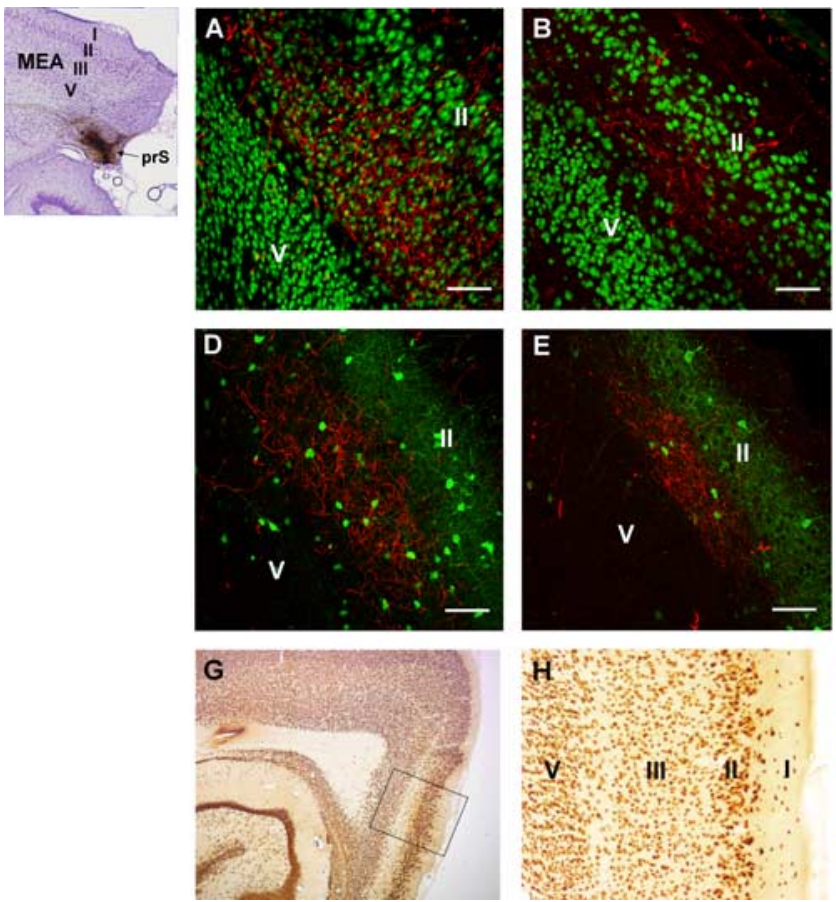

Figure 1. Projection from the prS to the degenerated MEA in chronic epileptic rats. The inset on the top left shows the injection spot of the anterograde tracer BDA in the superficial layers of the prS (horizontal section counterstained by Nissl staining). $\boldsymbol{A}, \boldsymbol{B}$, Confocal images of horizontal brain sections showing projecting fibers from the prS (red) in the MEA of a control rat $(\boldsymbol{A})$ and a chronic epileptic rat $(\boldsymbol{B})$ (6 months after KA-induced SE). Counterstaining with the neuronal marker NeuN (green) reveals the massive neuronal loss (and tissue shrinkage) in the MEA-III that is observed in most chronic epileptic rats. Presubicular fibers predominantly target the MEA-III in both the control and epileptic rats, despite the neurodegeneration in layer III in the epileptic rats. $\boldsymbol{C}$, Detail of the target area in the MEA-III from the chronic epileptic rat in $\boldsymbol{B}$. Counterstaining with DAPI (blue) reveals the presence of non-neuronal cells (possibly glia) in the neurodegenerated MEA-III that may be targeted by fibers from the prS. D-F, Same projections as shown in $\boldsymbol{A}-\boldsymbol{C}$, counterstained for the presence of PV (green) instead of NeuN. No difference was found in the amount of PV-positive neurons in the superficial layers of the MEA in chronic epileptic rats in comparison with controls (for details, see Results). PV-positive neurons are present in the target area from the prS in the MEA-III and -II in both control rats (D) and chronic epileptic rats $(\boldsymbol{E}, \boldsymbol{F})(\boldsymbol{F}$, counterstaining with DAPI in blue). $\mathbf{G}-\boldsymbol{I}$, Neuronal loss is extensive in layer III of the MEA in chronic KA rats also in the dorsal part of the MEA. G, NeuN-stained sagittal brain section showing the MEA around the level of recording in the dorsal part of the MEA from a chronic KA rat (Fig. $2 B$ ). $\boldsymbol{H}, \boldsymbol{I}$, Details of the MEA layers (rectangle in $\boldsymbol{G}$ ) of a control rat $(\boldsymbol{H})$ and a KA rat $(\boldsymbol{I})$, revealing extensive loss of neurons in layer III for the chronic KA rat. Scale bars: $\boldsymbol{A}, \boldsymbol{B}, \boldsymbol{D}, \boldsymbol{E}, 100 \mu \mathrm{m} ; \boldsymbol{C}, \boldsymbol{F}, 40 \mu \mathrm{m}$; (in $\boldsymbol{I}$ ) $H, I, 150 \mu \mathrm{m}$.

reaction was terminated by several rinses with Tris buffer and washing one time in $0.05 \mathrm{M}$ phosphate buffer. Sections were mounted onto gelatin-coated coverslips and air dried. Sections containing tracer were counterstained with a Nissl staining $(0.5 \%$ cresyl violet in $0.3 \%$ acetic acid). Next, sections were dehydrated through a series of alcohol solutions, placed in xylene, and coverslipped with Entellan.

For verification of the recording and stimulation locations in the electrophysiological experiments, sagittal sections containing the lesion marks were subjected to Nissl staining and inspected with a microscope.

A subset of the sections in both tracing and electrophysiological experiments were selected to be processed for neuronal nuclei (NeuN) or parvalbumin (PV) staining or a modified Timm's staining method (for details, see Sloviter, 1982). NeuN and parvalbumin staining were performed as described previously for NeuN (Tolner et al., 2003), using the monoclonal mouse anti-NeuN antibody [1:1000; monoclonal mouse anti-neuron-specific nuclear protein (NeuN) MAB377; Chemicon International, Harrow, UK] or the monoclonal mouse anti-parvalbumin antibody (PV) (1:10,000; p-3171; Sigma, Zwijndrecht, The Netherlands). Sections were photographed using bright-field illumination on an Olympus microscope and a digital camera (DP11; Olympus, Tokyo, Japan). Pictures were imported into Adobe Photoshop (version 6.0; Adobe Systems, San Jose, CA).

For quantification, sections used for immunocytochemistry were an-
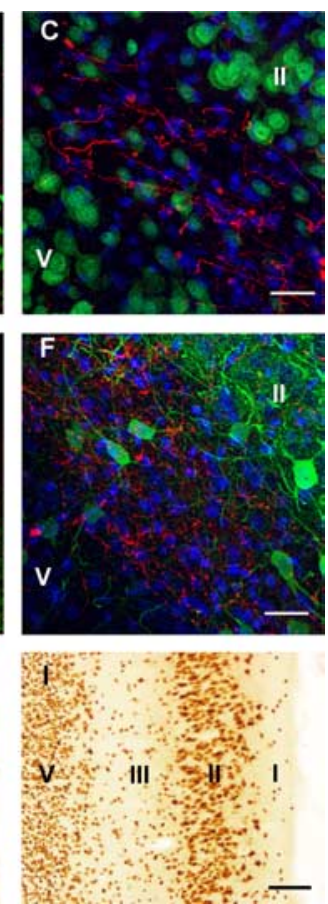

alyzed by an investigator unaware of the treatment group. Cell counting was performed as described in detail previously (Tolner et al., 2003). For the tracing experiments, the average number of neuronal profiles in NeuN- or PVstained sections in a given region for each animal was obtained by counting cells in two to three sections at the level of the tracer injection (in the prS) and at the level of the tracer projection (in the MEA). Cell counts were made of the superficial layers (II and III) in the PrS according to the description by Haug (1976) and Mulders et al. (1997). For the electrophysiological experiments, in each animal, the average number of neuronal profiles in the NeuN- or PVstained sections was based on cell counts made in two sections just medial or lateral to the recording site in the MEA. The density of detectable NeuN- or PV-immunoreactive neurons in the area of interest was assessed at $400 \times$ magnification using a microscopic grid $(30 \times 30$ $\mu \mathrm{m})$. To avoid including glial cells in the counts, we identified neurons as profiles $>7$ $\mu \mathrm{m}$. Boundaries of the MEA layers were determined according to the descriptions of Witter et al. (2000a). Thus, the neuron counts performed in this study are relative estimates of the amount of neurons at the level of the tracer injection or projection area, and immediately around the area in which the recordings were made. The neuronal loss in the MEA-III of the KA rats was accompanied by a concomitant shrinkage of the superficial MEA area in comparison with controls. Shrinkage of layer III was estimated by measuring the width of the layer at the level of the 16-channel probe. Statistical analysis between groups was performed using a Student's $t$ test (two-tailed distribution; twosample equal variance). The MEA neuronal cell densities of KA rats were calculated as a percentage of the average neuronal cell densities in control rats $(100 \%)$ and presented as mean \pm SEM. In Timm's stained preparations, the extent of synaptic reorganization of the mossy fibers (mossy fiber sprouting score) in the dorsal part of the hippocampus (from sagittal sections at the level of the stimulations) was evaluated by two observers according to a standardized $0-5$ scale according to the extent and density of zincstained granules (Cavazos et al., 1992).

Combined labeling of tracer and fluorescent immunohistochemistry. For combined labeling of the anterograde tracer and fluorescent immunostaining, a subset of free-floating sections from the traced animals was washed and incubated with either NeuN monoclonal antibody or PV monoclonal antibody as described above. Hereafter, sections were washed three times in PBS and incubated for $1.5 \mathrm{~h}$ at room temperature with streptavidin-Alexa Fluor 633 (dilution, 1:200; Invitrogen) and antimouse Alexa Fluor 488 (dilution, 1:200; Molecular Probes) in 0.05 м PBS plus $0.1 \%$ Triton X-100 plus $0.4 \%$ BSA. After three additional washes in PBS, sections were mounted on gelatin-coated slides and coverslipped using Vectashield mounting medium or Vectashield mounting medium with 4',6'-diamidino-2-phenylindole (DAPI) (UV excitation; Vector Laboratories). Images were acquired using a Zeiss LSM 510 confocal laser-scanning microscope (Carl Zeiss, Jena, Germany) and were processed using Zeiss LSM Image software.

Analysis of local field potentials. Off-line analysis was performed using Signal software (Cambridge Electronic Design, Cambridge, UK) and Matlab (Mathworks, Natick, MA) including both built-in and custommade functions. DC levels and low-frequency drift present in the recordings were removed by subtracting the best linear fit. One dimensional 
CSD was estimated by approximation of the second-order derivative in space using the following formula:

$$
\operatorname{CSD}(h, t)=\frac{\sigma_{h}(\Phi(h-n \Delta h, t)-2 \Phi(h, t)+\Phi(h+n \Delta h, t))}{(n \Delta h)^{2}} .
$$

$\operatorname{CSD}(h, t)$ is the current source density at fixed time $t$ and depth $h, \Phi(h, t)$ is the recorded potential at time $t$ and depth $h, \Delta h$ is the distance between recording sites $(100 \mu \mathrm{m})$, and $\sigma_{h}$ is the tissue conductivity, which was assumed to be constant. The parameter $n$ defines the amount of spatial smoothing applied to the data (Freeman and Nicholson, 1975; Ahrens and Freeman, 2001). In our study, we used a three-point CSD analysis $(n=1)$. CSD is presented in arbitrary units (in millivolts per square millimeter). To provide a crude estimate of the CSD at the most superficial recording site in the entorhinal cortex, a fictive site was introduced at which the local field potential was assumed to be equal to the most extreme recording site in entorhinal layer I. For quantification of the $n 3$ component, experiments were only taken into account for analysis if recordings included the adjacent source for the $\mathrm{n} 3$ component in a more superficial channel. Statistical analysis of quantified events in the CSD profiles was performed using a Mann-Whitney $U$ test (two-tailed distribution).

An important assumption that has to be made to allow the onedimensional CSD calculation is that the major extracellular current flows parallel to the recording track. We found that recordings made at slightly different angles with respect to the MEA layers or at different sites along the lateromedial direction displayed strong similarity. This indicates that the MEA domains that were activated by the presubicular stimulation occupied a rather wide extension of the entorhinal cortex. Consequently, it implies the absence of significant voltage gradients along the MEA in a direction parallel to the cortical surface, as was also shown before for subiculum and olfactory inputs to the entorhinal cortex (Van Groen et al., 1987; Kloosterman et al., 2003b).

To better characterize the dynamics of induced oscillations in the MEA, a wavelet analysis of the CSD signals (D'Attellis et al., 1997) was performed for both control and KA rats. We used a set of L2-normalized Gabor filters with apertures correlated to their wavelengths. Epochs displaying oscillations in the CSD profile of KA rats were analyzed, along with equivalent epochs of controls. The significance of the time-frequency amplitudes of a single measurement from KA rats was quantified using the following $z$-score: $Z=\left(G-\operatorname{mean}_{c}\left(G_{c}\right)\right) / \operatorname{std}_{c}\left(G_{c}\right)$, where $G$ is the (absolute value) Gabor amplitude of the epileptic trace and $G_{\mathrm{c}}$ are the Gabor amplitudes of the control traces.

\section{Results \\ Anatomical projection from the presubiculum to the MEA in chronic epileptic rats}

Because of the selective neurodegeneration in the MEA layer III in chronic epileptic rats, one might expect synaptic reorganization of the projections to this layer. To test this possibility, we analyzed the anatomical characteristics of the input to the MEAIII from the prS. The anterograde tracer BDA was successfully injected in the superficial layers (II and III) of the prS of control $(n=4)$ and chronic epileptic rats $(n=7)$.

Neuronal density was significantly decreased in layer III of the medial entorhinal area (MEA-III) for the chronic epileptic rats. For the KA rats, the number of remaining neurons in the MEA-III at the level of the tracer projection was assessed to be $39 \pm 11 \%$ $(n=7 ; p<0.001)$, in comparison with controls. For five of seven KA rats, neuronal loss in the MEA-III was extensive (ranging from 89 to $50 \%$ ), whereas two KA rats displayed less neuronal loss (34 and 11\%). In the KA rats with extensive neuronal loss in the MEA-III, considerable shrinkage of layer III was observed. Counterstaining with the nuclear marker DAPI (Sanna et al., 1992) revealed the presence of a large amount of non-neuronal cells in the degenerated part of the MEA-III (Fig. 1C), which likely are glia cells. Moreover, PV-positive interneurons in the
A

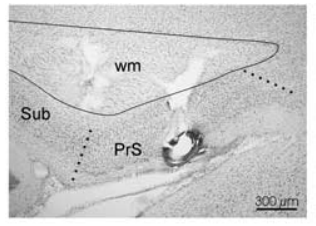

C
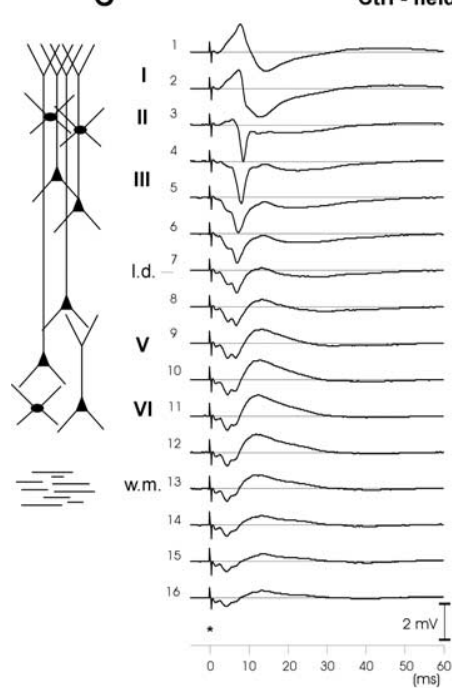

B

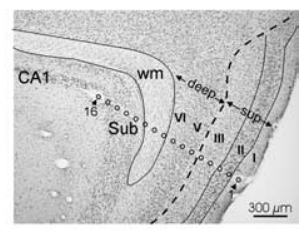

D

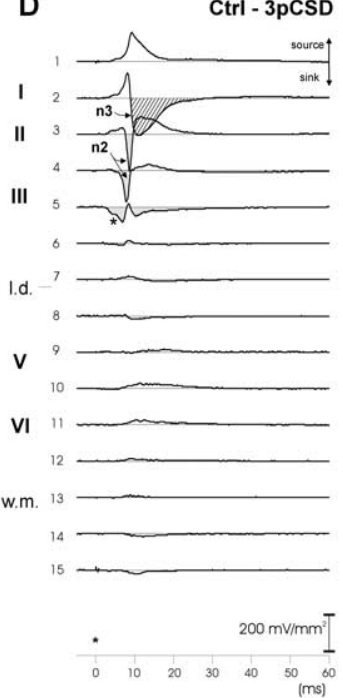

Figure 2. prS-evoked responses in the MEA of control rats. $\boldsymbol{A}$, Nissl-stained sagittal section of the parahippocampal formation illustrating the stimulation site in the $\mathrm{pr} S$. Dotted lines indicate the borders of the prS. $\boldsymbol{B}$, Nissl-stained sagittal section illustrating the recording track of a 16-channel silicon probe in the dorsal part of the MEA. Lesions were made at the 1st and 16th recording sites (arrowheads). Entorhinal layers are indicated with Roman numerals, with a separation between the deep layers (V and VI) and the superficial layers (I-III). The extent of the white matter and the borders of layer II are drawn as lines, and the lamina dissecans is drawn as a dashed line. $\boldsymbol{C}$, Typical example of laminar profiles of field potential responses (average of 4 sweeps) in the MEA evoked by prS stimulation (indicated by an asterisk) in control rats ( $n=8)$. The response to the test stimulus in the double-pulse protocol is shown. $D$, CSD laminar profile of the field responses shown in $\boldsymbol{A}$. Relevant sinks are shaded or striped, and the early negative components $\mathrm{n} 2$ and $\mathrm{n} 3$ in the superficial layers are indicated. The main effect of prS stimulation is seen in the occurrence of a sharp current sink (n2) that is of small amplitude in the MEA-III (indicated by an asterisk) and is most prominent in the MEA-II. The $n 2$ sink is followed by a broad current sink (n3 wave; striped) at the border of MEA-I and -II. See Results for details. The scheme of the layers of the entorhinal cortex on the left indicates the location of the recording sites, as reconstructed from histology. CA, Cornu ammonis; Ctrl, control; I.d., lamina dissecans; Sub, subiculum; sup, superficial; wm or w.m., white matter; 3pCSD, three-point CSD.

superficial layers of the MEA in KA rats are relatively spared as is shown in Figure $1 D-F$. No neuronal loss was observed in the prS.

The BDA injection sites were mostly confined to the superficial layers of the prS at dorsal and middle levels along the longitudinal axis of the prS in both groups of rats (Fig. 1, inset). In control animals, these injections gave rise to a bilateral plexus of labeled presubicular fibers that was most pronounced in the MEA-III (Fig. 1A). Despite the marked loss of neurons in layers III in most of the KA rats, the projection pattern of presubicular fibers was similar to that in controls (Fig. $1 B$ ). Apart from the main projection to the MEA-III, prS fibers also projected bilaterally to the MEA-I in both control and KA rats. A weaker projection was observed in the MEA-II. Outside the parahippocampal region, prS fibers were observed in the anteroventral and ventrolateral thalamic nuclei in both control and KA rats. In control rats, the observed pattern of termination of the prS fibers confirmed observations from previous anatomical studies (Kohler, 1984, 1985; Caballero-Bleda and Witter, 1993; Eid et al., 1996; van Haeften et al., 1997; Honda and Ishizuka, 2004). Because of 

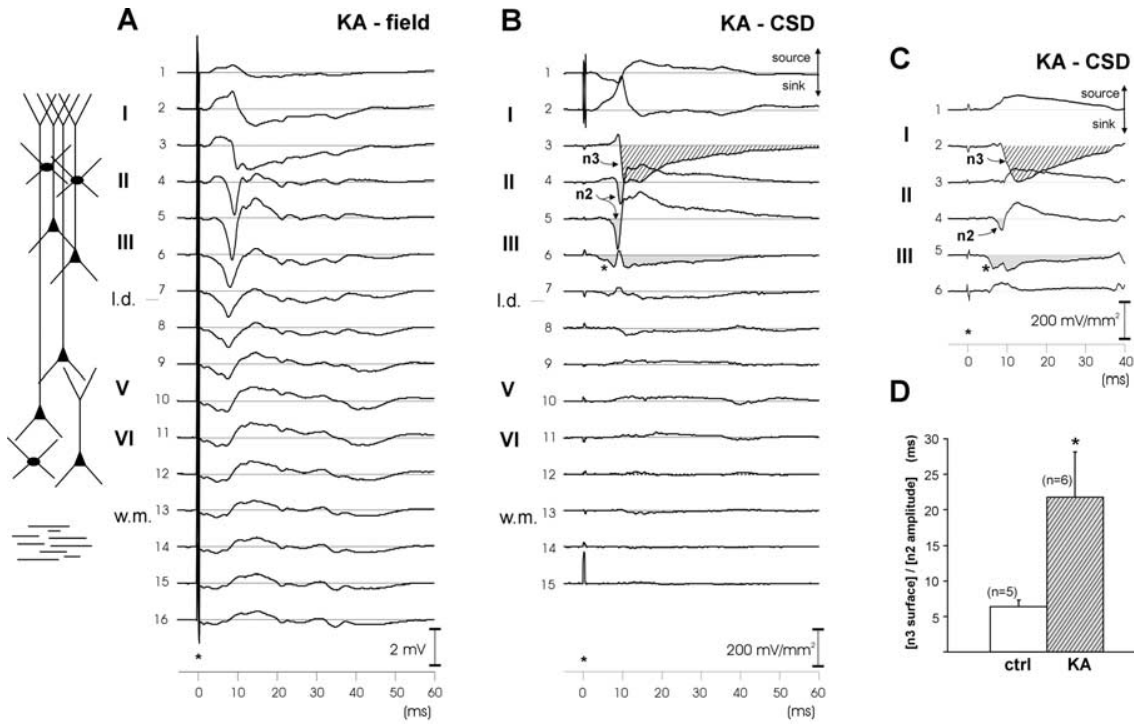

Figure 3. prS-evoked responses in the MEA of chronic epileptic rats. $A$, Typical example of the laminar profiles of field potential responses (average of 4 sweeps) in the MEA evoked by PrS stimulation (indicated by an asterisk) in a chronic epileptic rat ( 3 months after SE induction with KA, 30\% remaining neurons in the MEA-III). $\boldsymbol{B}$, CSD laminar profile of the field responses shown in $\boldsymbol{A}$. $\boldsymbol{C}$, Example of CSD laminar profile in a chronic epileptic rat (3 months after SE induction, 40\% remaining neurons in the MEA-III) displaying an $\mathrm{n} 2$ component of small amplitude. Relevant components are depicted as in Figure 2. See Results for details. $\boldsymbol{D}_{\text {, }}$ Comparison between the $\mathrm{n} 3$ current sink (wave) at the edge of the MEA-I and $-I I$ in controls and chronic epileptic rats. The ratio between the $\mathrm{n} 3$ and $\mathrm{n} 2$ component was significantly larger for chronic epileptic rats $(n=6)$ in comparison with controls $(n=5$; $p=0.026$; Mann-Whitney $U$ test). The asterisk indicates early sink that is evoked in MEA-III after prS stimulation. Error bars indicate SE. ctrl, Control; I.d., lamina dissecans; w.m., white matter.
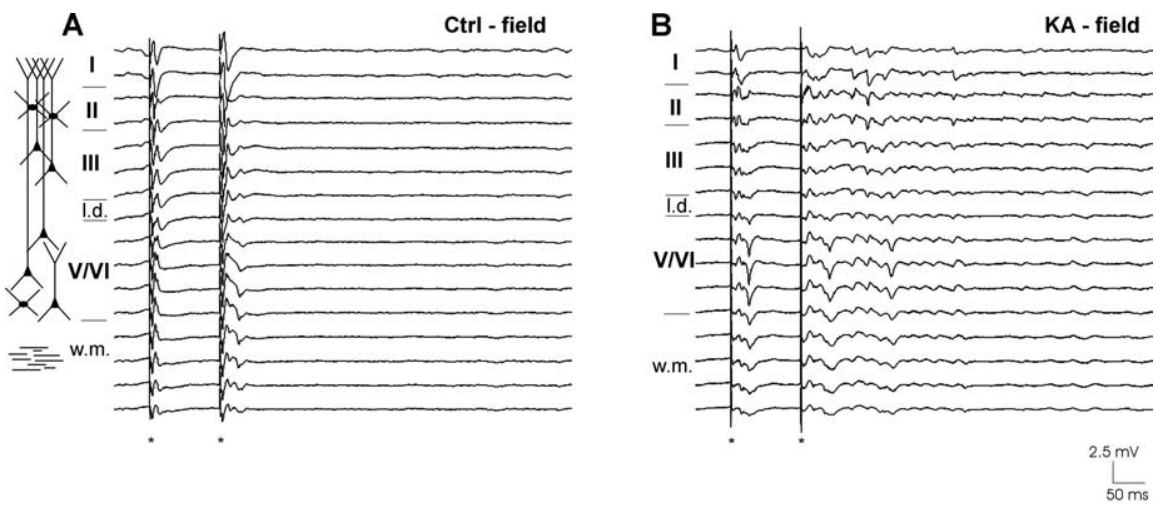

Figure 4. prSstimulation evokes oscillations in the MEA of chronic KA rats. Typical examples of laminar profiles of field potential responses (nonaveraged) in the MEA evoked by prS stimulation (indicated by an asterisk) in a control rat $(\boldsymbol{A})$ and a chronic KA rat (B) (4 months after KA-induced SE, 40\% remaining neurons in the MEA-III). The indication of the MEA layers is as in Figures 2 and 3. Ctrl, Control; I.d., lamina dissecans; w.m., white matter.

the nonquantitative character of the tracing method, it cannot be determined whether the density of the prS-MEA connection was altered in the chronic KA rats. However, we can conclude that the prS projections are still present in chronic KA rats but that they likely have changed their target because a large number of MEAIII neurons have disappeared.

Functional alterations in the presubiculum-MEA connection To identify whether the functionality of the observed prS projections was altered in the chronic epileptic rat model, the physiological characteristics of the prS-MEA connection were examined in vivo for both control and chronic KA rats.

Histology of the (para)hippocampal region in the recorded rats For KA rats that were studied electrophysiologically $(n=6)$, the amount of remaining neurons in the MEA close to the area of

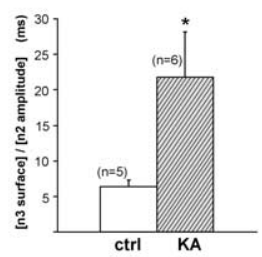

recording was assessed to be $100 \pm 7 \%$ for MEA-II, $53 \pm 10 \%$ for MEA-III $(p<$ $0.001)$, and $110 \pm 4 \%$ for MEA-V/VI, in comparison with controls $(n=8)$ (Fig. $1 G-I)$. Regarding layer III, five of six KA rats displayed $>50 \%$ loss in the MEA-III, whereas one KA rat displayed no detectable MEA-III loss.

To get an estimate of possible alterations of the inhibitory network in the superficial layers of the MEA, we assessed the amount of PV-positive neurons in the superficial layers of the MEA that surrounded the recorded area. In KA rats, the density of PV-positive neurons in superficial MEA was not altered for the MEA-II in comparison with controls $(102 \pm 7 \% ; n=6 \mathrm{KA}, n=8$ controls) but was significantly increased in the MEA-III $(134 \pm 10 \%, p=0.02)$. The MEA-III layer width was diminished by $28 \pm 3 \%(p<0.05)$ in chronic KA rats compared with controls, so that the increase in PV density in the MEA-III can be related to shrinkage of this layer and is not attributable to an absolute increase of PV neurons in layer III. In addition, we investigated the amount of hippocampal reorganization using Timm's staining to visualize zinc. Five KA rats displayed robust mossy fiber sprouting (Timm's score of 3-5). These KA-treated rats displayed $>50 \%$ MEAIII loss and were observed to have spontaneous seizures, starting from $\sim 2$ weeks after KA-induced SE. The one KA rat that displayed no detectable neuronal loss had a Timm's score of 2-3. This rat had not been observed to display spontaneous seizures, although this cannot be excluded, because continuous video monitoring was not performed.

In the eight control and six KA rats that were recorded with a 16-channel probe, histological verification of the recording track showed that recordings were performed in all layers of the MEA (Fig. $2 B$ ). In those rats in which recordings were performed in hippocampus as well (six control rats and three KA rats), the hippocampal recording track covered area CA1 and the upper blade of the DG (see Fig. 7C).

\section{Characterization of double-pulse responses to presubiculum} stimulation in control rats

Stimulation of the dorsal prS evoked short-latency and in some cases long-latency field responses in the MEA of control rats $(n=$ 8 rats) (Fig. $2 C$ ). To be able to identify the local generators of these events, we performed CSD analysis.

The short-latency responses evoked by prS stimulation were confined to the superficial layers (I-III) of the MEA (Fig. 2D), as revealed by CSD analysis. In four of eight control experiments, a first small negative deflection of short latency was found in the MEA-II, corresponding to a transient sink in the MEA-II flanked 
by sources in the MEA-II and MEA-III (n1; not seen in the example of Fig. 2). This negative deflection is thought to represent an antidromic spike caused by activation of passing perforant path fibers. This is supported by the following: (1) the short latency of the deflection, (2) the minimal time jitter at different stimulus intensities, and (3) the absence of pairedpulse facilitation (double-pulse stimulation with $100 \mathrm{~ms}$ interpulse interval). The first event observed after this antidromic response was a negative-going deflection starting at $\sim 4 \mathrm{~ms}$, corresponding to a slowly increasing sink that first appeared in the MEA-III [Fig. 2D, asterisk in channel 5 (ch5)]. At the same time, a source is apparent in the MEA-II near the edge with the MEA-I (Fig. 2D, ch3). This initial current sink in the MEA-III is likely caused by monosynaptic connections from the prS with principal neurons in the MEA-III and dendrites of the MEA-II extending into layer III. Shortly after the small initial sink in layer III, a larger and sharper sink becomes apparent in layers III and II. We refer to this negative peak in the CSD profile as the $n 2$ sink. The latency of the $n 2$ sink increased from layer III to layer II (e.g., in the example of Fig. $2 D$, peak times were $6.8,7.8$, and $8.5 \mathrm{~ms}$ for channels 5,4 , and 3 , respectively). At the border of layers $\mathrm{I} / \mathrm{II}$, the $\mathrm{n} 2$ sink was associated with a transient source (Fig. 2D, ch2). These fast transients are interpreted to reflect the generation and (back)propagation of action potentials. Additionally, it is not unlikely that two populations of neurons, one with somata in the MEA-III and the other one in the MEA-II, are activated by the prS inputs, the former being ahead of the latter by a little less than $1 \mathrm{~ms}$. Although presubicular fibers are known to form synapses directly with cells of layer III and to a lesser extent with cells of layer II (Kohler, 1984, 1985; Caballero-Bleda and Witter, 1993; van Haeften et al., 1997), the observed CSD profile of the prSevoked responses in control rats points to stronger activation toward layer II, and not layer III. We think that the biophysics of the CSD provides an explanation for this: in contrast to layer II cells, the somata of layer III neurons do not have a very compact distribution. Thus, the synaptic presubicular input will be much more dispersed in layer III; because of the more compact organization of layer II, a less dense input could give rise to somewhat stronger fields (current densities) in this layer. Alternatively, it has been shown by van Haeften et al. (1997) that projections to layer III exert both excitatory and inhibitory effects. Therefore, the net synaptic current in layer III may be of small magnitude or may not even be detected, because currents of opposite signs cancel each other out. A similar response after PrS stimulation was recently described in guinea pigs (Bartesaghi et al., 2005).

Directly after these events, a prolonged current sink with small amplitude was observed in the MEA-III (Fig. $2 D$, ch5) in six of eight controls that could last $\sim 30-50 \mathrm{~ms}$. After the n2 component in the MEA-II/III, a late large sink (n3 "wave") with slow kinetics can be observed at the edge of layers I and II (Fig. 2D, ch2), which is flanked by a source in the superficial
B

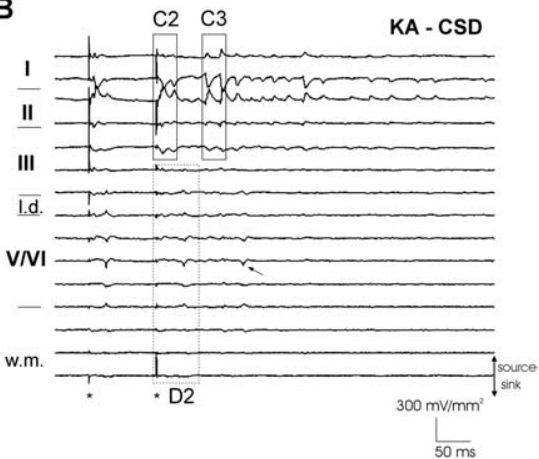

D1

D2

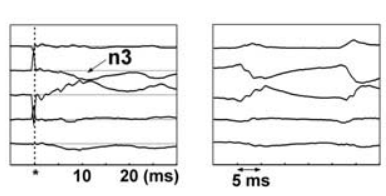

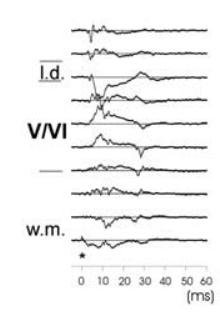

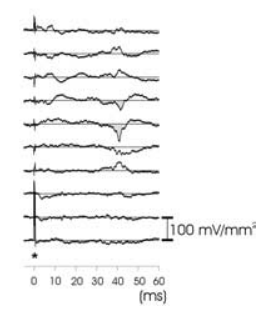

Figure 5. CSD laminar profiles of the field responses shown in Figure 4. Both in control rats $(\boldsymbol{A})$ and in KA rats $(\boldsymbol{B})$, prS The relevant current sinks are shaded gray. Ctrl, Control; I.d., lamina dissecans; w.m., white matter. Asterisks in A, B, C1, C2, D1, and $\mathbf{D} 2$ represent time of $\mathrm{pr}$ stimulation.

MEA-II (Fig. 2D, ch3) and in the MEA-I (Fig. 2D, ch1). This sink appears at a latency of $\sim 9 \mathrm{~ms}$ and lasts $\sim 30 \mathrm{~ms}$ and is likely the result of disynaptic and/or polysynaptic activation of superficial neurons.

Using a double-pulse protocol with an interstimulus interval of $100 \mathrm{~ms}$, we found that, in all cases, the short-latency responses after the test stimulus were larger than the response to the conditioned stimulus, showing the existence of paired-pulse facilitation and supporting the synaptic origin of the responses (see Figs. 4A, 5A).

The long-latency field response consisted of a population spike in deep layers (latency, 26-36 ms after the prS stimulus) and a wave-like negativity in the MEA-III. The long-latency response was not observed in all experiments, and usually it was seen only after the test stimulus in a paired-pulse protocol at the largest stimulus intensity used of $999 \mu \mathrm{A}$ (four of eight rats) (not seen in the example of Fig. 2, but see Fig. 5D1). The response is interpreted to be the result of stimulation of perforant path fibers that pass through the prS and are likely activated with prS stimulation (Witter and Amaral, 2004). This is in line with observations of similar responses that were evoked by subiculum stimulation (for details, see Kloosterman et al., 2003b; Tolner et al., 2005). Direct activation of the hippocampus by the prS (Witter and Amaral, 2004) could also explain the presence of a longlatency response, because we did not observe a strict relationship between the occurrence of a late response and the presence of an antidromic spike in the MEA-II.

Altered responses to prS stimulation in chronic epileptic rats In chronic epileptic rats, stimulation of the dorsal prS also evoked both short- and long-latency field responses in the MEA $(n=6$ 
Table 1. Latencies of the prS-evoked responses in superficial layers of the MEA as recorded in vivo in eight control and six chronic epileptic rats at 2-4 months after induction of SE with KA

\begin{tabular}{|c|c|c|c|c|c|}
\hline & $\begin{array}{l}\text { Latency onset } \\
\text { (ms) }\end{array}$ & $\begin{array}{l}\text { Latency peak } \\
\text { (ms) }\end{array}$ & $\begin{array}{l}\text { Peak amplitude } \\
\left(\mathrm{mV} / \mathrm{mm}^{2}\right)\end{array}$ & $\begin{array}{l}\text { Duration } \\
\text { (ms) }\end{array}$ & $\begin{array}{l}\text { Area under curve } \\
\left(\mathrm{ms} \times \mathrm{mV} / \mathrm{mm}^{2}\right)\end{array}$ \\
\hline \multicolumn{6}{|l|}{ Control } \\
\hline $\mathrm{n} 1(4: 8)$ & $1.08 \pm 0.18$ & $1.75 \pm 0.29$ & & & \\
\hline Negative deflection MEA-III (asterisk in Fig. 3D; 8:8) & $3.80 \pm 0.52$ & $6.82 \pm 0.51$ & & & \\
\hline $\mathrm{n} 2$ (observed 8:8; measured 6:8) & & $8.74 \pm 0.70$ & $234 \pm 42$ & & \\
\hline n3 (observed 8:8; measured 5:8) & $9.04 \pm 0.60$ & & & $24.36 \pm 2.27$ & $1479 \pm 279$ \\
\hline \multicolumn{6}{|l|}{ Epileptic } \\
\hline $\mathrm{n} 1(3: 6)$ & $0.97 \pm 0.04$ & $1.53 \pm 0.04$ & & & \\
\hline Negative deflection MEA-III (asterisk in Fig. 4B;6:6) & $3.64 \pm 0.54$ & $7.79 \pm 0.56$ & & & \\
\hline $\mathrm{n} 2(6: 6)$ & & $8.71 \pm 0.59$ & $84 \pm 16^{*}$ & & \\
\hline n3 $(6: 6)$ & $7.5 \pm 0.61$ & & & $25.99 \pm 6.07$ & $1517 \pm 507$ \\
\hline
\end{tabular}

Latencies of events were calculated from the three-point CSD analysis of the data. Responses were measured at the stimulus intensity that yielded the maximal response. Values are means \pm SEM.

${ }^{*} p<0.01$ (compared with controls; Mann-Whitney U test).
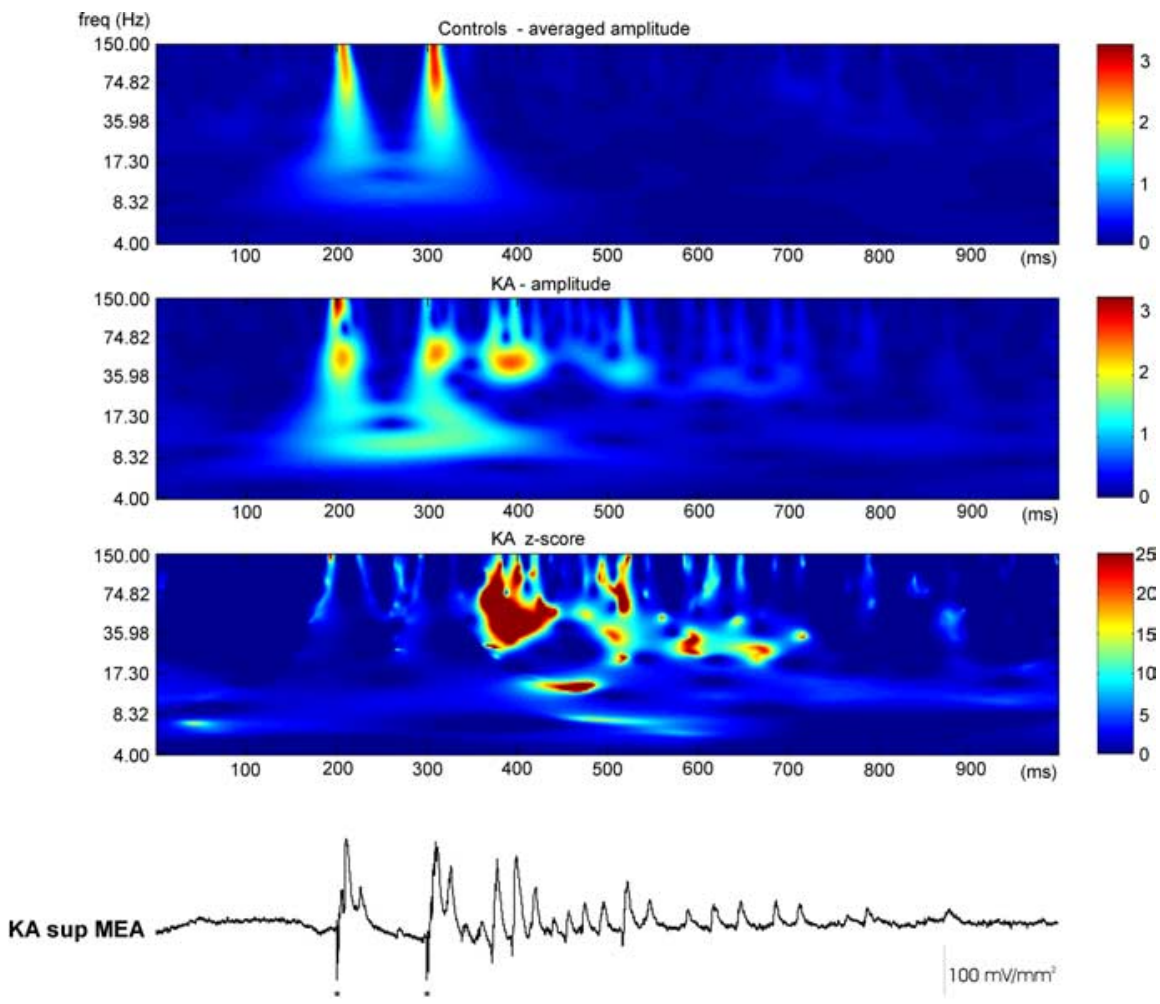

Figure 6. Wavelet analysis of the oscillations in channel 3 in the CSD laminar profile of the $K A$ rat from Figures $4 B$ and $5 B$, revealing the time-frequency characteristics of the superficial oscillations. Shown is the time-frequency $z$-score Gabor amplitude of the single-channel CSD during paired-pulse stimulation in the prS. The pulses were delivered at 100 and $200 \mathrm{~ms}$ relative to the beginning of the plot (horizontal axis) in one epileptic and five control animals. The current source densities of the second "superficial" channel were convoluted with a set of Gabor filters of logarithmically increasing frequencies (vertical axis) ranging from 4 to $150 \mathrm{~Hz}$. The $z$-score, represented in pseudo-color coding, was computed as described in Materials and Methods using five control traces. The averaging, the SD, and the subtraction were performed independently for each time-frequency point. In this example, after prS double-pulse stimulation (indicated by the 2 asterisks at a $100 \mathrm{~ms}$ interval) in the KA rat, superficial-layer oscillations are evoked that are more or less continuous for $\sim 300 \mathrm{~ms}$ in the range of $20-50 \mathrm{~Hz}$. freq, Frequency; sup, superficial.

KA rats) (Fig. $3 A, B$ ). The short-latency responses to prS stimulation in epileptic rats did not present differences in latency when compared with the control responses, as can be seen from the data in Table 1 . The peak amplitude of the $\mathrm{n} 2$ component, however, was significantly decreased in KA rats in comparison with controls (Table $1 ; p<0.01$ ). The long-latency field response that was seen only in some control recordings was observed in five of six KA rats (latency of deep-layer population spike, 26-40 ms after stimulation) (it was of small amplitude in the example of Fig. 3, but see Fig. 5D2). In contrast to controls, the late response was apparent both after the conditioning (five of six rats) and the test stimulus (four of six rats) in a paired-pulse protocol and occurred at a wider range of stimulus intensities. The size, shape, and location of this late response did not differ from that in control rats.

The main significant difference between control and KA rats, however, was found in the ratio between the $\mathrm{n} 3$ wave and the $\mathrm{n} 2$ component. Although the $\mathrm{n} 2 \mathrm{com}-$ ponent was evident in all animals, it had a small amplitude in five of six KA rats (Fig. $3 C$ ), including the one $\mathrm{KA}$ rat without MEA-III loss. Even in the cases of a small n2 component, the n3 component was very prominent (Fig. $3 B, C$ ). To compare the ratio between $\mathrm{n} 3$ and $\mathrm{n} 2$ components for control and KA rats, the area under the curve of $n 3$ was divided by the amplitude of the $\mathrm{n} 2$ largest sink (Table 1). This ratio was significantly larger in KA rats as shown in Figure 3D $(p<0.026)$.

Oscillations in the beta/gamma frequency range occur in superficial layers of the MEA after double-pulse stimulation of the presubiculum in chronic epileptic rats In contrast to control rats, in which responses returned to baseline after doublepulse prS stimulation (Fig. $4 A$ ), the responses were followed by afterdischarges in the form of oscillatory potentials in all KA rats that exhibited extensive neuronal loss in the MEA-III ( $n=5$ of 6 ). These oscillations were observed shortly after the response to the test stimulus and had a variable duration (between 200 and $600 \mathrm{~ms}$ ) (Fig. 4B). CSD analysis revealed that these oscillations were confined to the superficial layers of the MEA (Fig. $5 B$ ). Oscillations were never observed in control rats $(n=8)$ and were not observed in the KA rat that did not display neuronal 
loss in the MEA-III. There was no apparent relationship between the stimulus intensity and the occurrence of oscillations. All KA rats displaying superficial oscillations showed a long-latency response to prS stimulation, consisting in a sharp current sink in the MEA-V, followed by a smaller and broader sink in the MEA-III (Fig. 5D2). However, a direct link between the long-latency components and the superficial oscillations was not apparent. Superficial oscillations occurred both in combination with weak and with strong long-latency responses, and could be absent in cases with a strong long-latency component. The configuration of sinks and sources of the superficial oscillations resembled that of the $\mathrm{n} 3$ wave of the shortlatency response to prS stimulation (Fig. $5 C 1-C 3$ ). In one KA rat (example of Fig. $5)$, high-frequency activity $(\sim 400 \mathrm{~Hz}$ ripple) was observed at the start of the source that was observed in the MEA-II both during the short-latency prS response and at the start of the oscillations (Fig. 5B, ch3;C2-C3). The superficial oscillations consisted of current sinks that were observed in the MEA-I and in some cases also in the MEA-III, with accompanying current sources observed in the MEA-II. The time course of the superficial oscillations was quantified by way of time-frequency analysis as shown in Figure 6. The different KA rats displayed oscillations confined to the superficial layers of the MEA with variable main frequencies but always within the $\beta / \gamma$ frequency range $(20-100 \mathrm{~Hz})$.

In three of five cases in which superficial oscillations were observed after double-pulse stimulation of the prS, additional events were present in deep layers of the MEA. A series of current sinks was observed in layers $\mathrm{V}$ and VI, occurring with a frequency of $6-8 \mathrm{~Hz}$ (Fig. $7 A$, bottom boxed area). Deep-layer events showed a phase relationship with events in the subiculum (Fig. $7 A$, recording in one of the three rats) and DG (Fig. $7 A$, recordings in two of the three rats; $B$ ). Event-related overlay of the signals, triggered on the initial transient in deep layers (Fig. $7 B$ ) or on the peak of the superficial oscillations (Fig. 7C) did not reveal phase-locked events in either the superficial or deep layers, respectively.

\section{Discussion}

The present results demonstrate that rats with a chronic epileptic condition show physiological alterations that are most prominent in the superficial layers of the MEA. The occurrence of alterations correlated with marked neurodegeneration in layer III of the MEA. First, it was shown that the anatomical input from the presubiculum to the MEA-III was still present in chronic KA rats. Second, stimulation of the presubicular input in KA rats revealed a decreased amplitude of the population spikes in layers III and II and of the $\mathrm{n} 2$ current sink, whereas the $\mathrm{n} 3$ wave (at the border of layers I and II) was still very prominent. Third, there
B

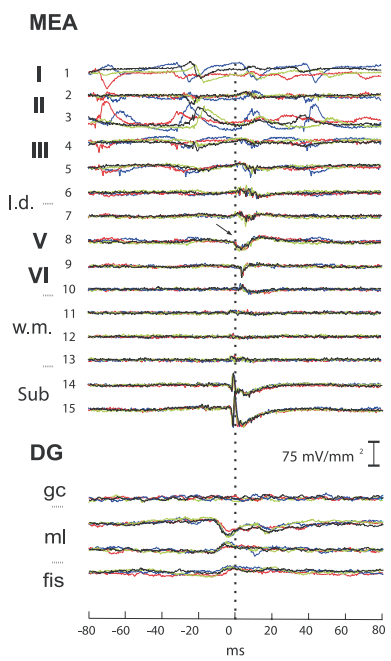

C

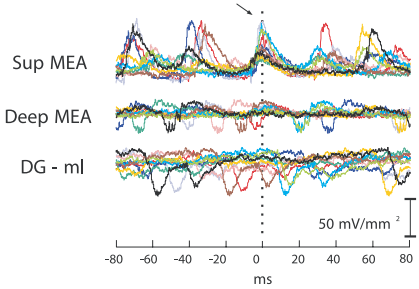

D

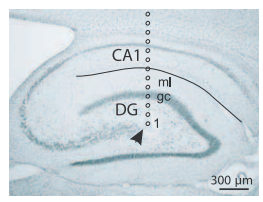

Figure 7. Oscillations occur occasionally in deep layers of the MEA after double-pulse stimulation of the prS in chronic epileptic A typical example of CSD laminar profiles of field potential responses (nonaveraged) in the MEA evoked by prS stimulation a asterisk) in a chronic KA rat (3.5 months after SE induction with KA, 50\% remaining neurons in the MEA-III). The MEA layers, in the deep MEA some events were observed that were in the theta range $(\sim 8 \mathrm{~Hz})$. These deep-layer events were synchronous with events recorded in the $D G$ and subiculum. $B, C$, Overlays of the events triggered at $\boldsymbol{B}$, the start of the current sink observed in the MEA-V (arrow in channel 8), and at $C$, the peak of the wave in the MEA-II (arrow in the superficial MEA trace), did

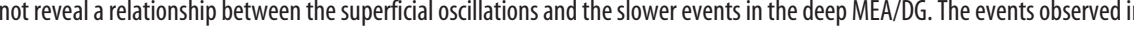
the deep MEA, however, are in phase with events in the DG and subiculum. The short time delay between the occurrence of these 列 events observed in channel 3 (see top boxed area in $\boldsymbol{A}$ ): the deep MEA taken at channel 8 . D. Histology of the recording locations in the hippocampus. The Niss-stained sagittal section illustrates the recording track of a 16 -channel silicon probe in the dorsal part of the hippocampus. Lesions were made at the 1st (arrowhead) and 16th recording sites. CA, Cornu ammonis; gc, granule cell layer; ml, molecular layer; fis, fissure; I.d., lamina dissecans; Sub, subiculum; Sup, superficial; w.m., white matter.

was the emergence of afterdischarges in the form of oscillatory activity $(20-100 \mathrm{~Hz})$ localized in superficial layers. Moreover, our in vivo observations revealed a dissociation between the deep and superficial layers with respect to induced oscillatory activity in chronic epileptic rats.

\section{Targets for the prS in a degenerated MEA-III}

Presubicular fibers appeared normally distributed in the MEA in chronic epileptic rats, despite neurodegeneration in the MEA-III. Our observation that the number of PV-positive neurons was not altered in KA rats suggests a preservation of this type of interneurons in the superficial layers of the MEA. This is the most prominent type of inhibitory interneuron in the superficial entorhinal cortex where it forms a very dense plexus around layer II neurons (Wouterlood, 2002). The view that interneurons are preserved in superficial MEA after epilepsy is supported by observations in different rat models for epilepsy (Du et al., 1995; Eid et al., 1999; Kobayashi et al., 2003; van Vliet et al., 2004). With the loss of mainly principal neurons in the MEA-III, predominant targets of the prS in chronic KA rats are likely to be the following: PV-positive interneurons in superficial MEA (Fig. 8, 1) (Wouterlood et al., 1995), 


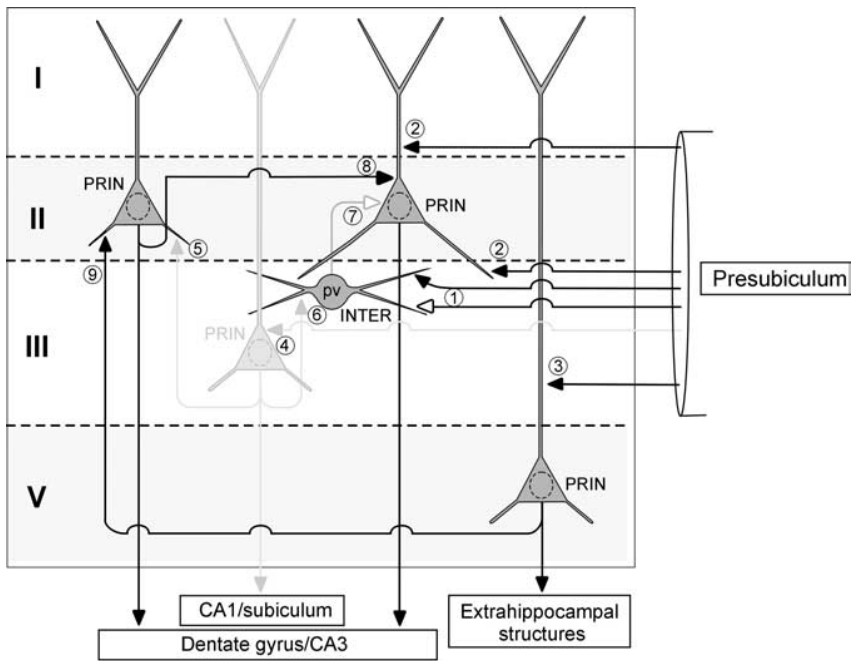

Figure 8. Scheme that summarizes the possible connections with and within the superficial layers of the MEA of chronic epileptic rats with regard to input from the prS. Cells are depicted as principal neuron (PRIN) or interneuron (INTER) (PV, parvalbumin-positive). $\bullet$, Excitatory synaptic connections; $\triangleright$, inhibitory synaptic connections. After chronic epilepsy in the KA model, neuronal loss affects mainly principal neurons in the MEA-III (gray shading indicating loss). Targets of the prS in the MEA in KA rats are presumed to be as follows. 1, Interneurons in the superficial MEA [targeted by excitatory and to a lesser extent by inhibitory presubicular fibers (van Haeften et al., 1997)]. 2, Dendrites of MEA-II neurons in the MEA-III and to a lesser extent in the MEA-I (Caballero-Bleda and Witter, 1994). 3, Dendrites of MEA-V neurons in the MEA-III (Wouterlood et al., 2004). 4, Remaining principal neurons in the MEA-III (Caballero-Bleda and Witter, 1994). 5, Connections between MEA-III and MEA-II neurons may be involved in the direct response to prS stimulation in the superficial MEA. 6, With loss of mainly principal neurons, PV-positive interneurons that control inhibition in layers II and III receive less activation after prS stimulation and may even be inhibited. 7, 8, This would result in decreased inhibition (7) and increased excitability among remaining principal neurons (8) (Eid et al., 1999; van Vliet et al., 2004; the present study). 9, Connections between deep and superficial neurons (Kloosterman et al., 2003b; van Haeften et al., 2003) might add to the hyperexcitability of the superficial layers. This basic scheme is adapted from Witter and Amaral (2004).

MEA-II neurons (Fig. 8, 2) (Kohler, 1985; Caballero-Bleda and Witter, 1993), and dendrites of the MEA-V neurons (Fig. 8, 3) (Wouterlood et al., 2004). For the latter possibility, however, we did not find direct electrophysiological evidence.

\section{Interpretation of the direct prS-evoked responses in the superficial MEA in control versus KA rats}

Most epileptic rats displayed $>50 \%$ loss of MEA-III neurons. Nevertheless, the direct response to prS stimulation in the MEAIII had a similar pattern to that in control rats, consisting of a strong response ( $\mathrm{n} 2$ component) in the MEA-II. How can this be explained? Given that the anatomical projection of the prS targets the MEA-III in both control and KA rats, activation of the MEA-II could be the result of presubicular fibers targeting dendrites of layer II neurons that extend into layer III, a connection that has been described for control rats (Caballero-Bleda and Witter, 1994). In addition, the MEA-II could get activated by an indirect input via the remaining neurons in layer III (Fig. 8, 4, 5). The latter assumes a close connection between the MEA-III and MEA-II principal cells. Anatomical studies suggest the existence of connections between ECIII and ECII principal cells (Kohler, 1986; Germroth et al., 1989). Stimulation of the dorsal psalterium (carrying fibers from the prS) in guinea pig revealed functional connectivity between principal cells from ECIII toward ECII (Bartesaghi et al., 1988). The observation that the $\mathrm{n} 2$ component was decreased in most of the KA rats could be explained by the loss of the MEA-III neurons. The localization of the $\mathrm{n} 2$ compo- nent at the edge of the MEA-II and -III, strengthens the view that sinks are actively generated in dendritic compartments of MEA-II or MEA-III neurons. We interpret the n3 wave in the MEA-I/II to reflect EPSCs at the level of MEA-II apical dendrites possibly caused by monosynaptic and/or polysynaptic activation among MEA-II neurons. MEA-II neurons that activated during the $\mathrm{n} 2$ component would subsequently activate other MEA-II neurons, leading to the firing of these neurons (Fig. 8, 8). Recurrent connections among layer II principal cells, however, have not been detected in vitro (Dhillon and Jones, 2000). Clearly, more detailed studies are needed for a complete understanding of the functional connections among MEA-II principal neurons.

The most prominent electrophysiological difference between $\mathrm{KA}$ and control rats was that, although the $\mathrm{n} 2$ sink was decreased in amplitude for the KA rats, the $\mathrm{n} 3$ wave after $\mathrm{n} 2$ was as broad as in controls and did not decrease in amplitude. We interpret these findings as follows: loss of MEA-III principal cells will leave superficial interneurons dormant as previously suggested by Eid et al. (1999) and van Vliet et al. (2004) (Fig. 8, 6). Because PVpositive cells in layers III and II form a dense plexus around layer II principal neurons (Wouterlood et al., 1995), this will result in loss of inhibition of remaining principal cells in the MEA-II and -III (Fig. 8, 7). Furthermore, the inhibitory interneuron population in the superficial MEA receives, in addition to excitatory inputs from surrounding pyramidal cells, presubicular input, 20-30\% of which is GABAergic (van Haeften et al., 1997) (Fig. 8, 1). With loss of excitatory input from MEA-III principal cells, the inhibitory drive onto superficial-layer interneurons (by the GABAergic fibers from the prS) will be relatively larger than in controls, leading to disinhibition. Preliminary results from in vitro intracellular recordings in slices of chronic KA rats reveal that prS stimulation evokes prolonged depolarization in both MEA-II and MEA-III neurons, corresponding to the $\mathrm{n} 3$ wave seen in vivo (Tolner et al., 2004).

\section{Possible mechanisms underlying superficial oscillations}

During the oscillations in superficial layers, a sink-source configuration in the MEA-I/MEA-II was observed that was similar to that of the transient prS-evoked response. The same pattern was seen in superficial oscillations, induced by subiculum stimulation in chronic KA rats (Tolner et al., 2005). This suggests that similar processes underlie both the short-latency prS response and the oscillations and that the oscillations are generated locally at the level of the MEA-II apical dendrites. However, given the functional connectivity that exists between the deep and superficial MEA (Jones and Lambert, 1990; Bear et al., 1996; Lopantsev and Avoli, 1998; Stewart, 1999; Kloosterman et al., 2003a), it is possible that deep-superficial layer activity contributes to the occurrence of superficial-layer oscillations (Fig. 8, 9). Diminished control of interneuron activity could lower the threshold for excitation of MEA-II neurons, resulting in oscillations. Nevertheless, we cannot exclude the possibility that interneurons are involved in the occurrence of the superficial oscillations. The observation of highfrequency activity in the MEA-II in one of our KA rats supports this possibility, because high-frequency activity is presumed to correlate with firing of interneurons (Bragin et al., 1999). Additionally, altered cholinergic control of entorhinal activity may contribute to the occurrence of superficiallayer oscillations (Dickson and Alonso, 1997; Gloveli et al., 1999; van der Linden et al., 1999).

Specific properties of superficial entorhinal neurons could be altered in chronic KA rats, adding to stronger excitability in the superficial MEA (Alonso and Llinas, 1989; Magistretti et al., 1999; 
Dickson et al., 2000). However, basic properties of superficial EC neurons in chronic epileptic rats do not appear to be altered (Bear et al., 1996; Kobayashi et al., 2003). Additional studies at the cellular level are needed to determine more precisely which mechanisms contribute to generation of superficial oscillations in chronic KA rats.

\section{Dissociation between the deep and superficial MEA layers in chronic KA rats}

Deep-layer oscillations occurred also in KA rats, but at a lower frequency than the superficial-layer oscillations, indicating that the networks of deep and superficial layers differ in their dynamical properties. The fact that deep-layer events were synchronous with events in the hippocampus suggests that the hippocampal neuronal network participates in the deep-MEA oscillations. Based on direct connectivity between deep EC and the dentate gyrus (Dugladze et al., 2001; Gloveli et al., 2001), reverberation of activity might occur between the deep EC and the hippocampus.

Deep-layer cells show different physiological properties than superficial-layer cells (Alonso, 2002). In addition, the inhibitory circuitry of superficial layers appears to be more substantial compared with that of deep layers (Jones and Heinemann, 1988; Jones, 1994; Wouterlood et al., 1995; Funahashi and Stewart, 1998; Woodhall et al., 2005). These different properties can account for the differences in oscillatory behavior. In our experiments, it is not clear whether the occurrence of superficial oscillations is independent from the deep-layer oscillations in chronic epileptic rats, although this appears to be the case on the basis of the event-related averages presented in Figure 7, $B$ and $C$.

Different kinds of ictal-onset patterns in EC and hippocampus have been reported in epileptic rats injected intrahippocampally with kainate (Bragin et al., 1999). Spencer and Spencer (1994) noted that spiking onset in TLE patients was not related to activity of the EC but was of hippocampal origin. A similar distinction was made by Bartolomei et al. (2004), showing that ictal onsets with hypersynchronous spiking activity originated in the hippocampus and low-voltage fast ictal onsets in the EC. Together, these findings indicate that an extensive network within the mesial temporal lobe, including the MEA and hippocampal formation, undergoes dynamical changes in epileptic rats and likely also in human patients. These changes would be manifested as different kinds of oscillations, the characteristics of which depend on local properties of particular subareas.

\section{References}

Ahrens KF, Freeman WJ (2001) Response dynamics of entorhinal cortex in awake, anesthetized, and bulbotomized rats. Brain Res 911:193-202.

Alonso A, Llinas RR (1989) Subthreshold $\mathrm{Na}^{+}$-dependent theta-like rhythmicity in stellate cells of entorhinal cortex layer II. Nature 342:175-177.

Alonso AA (2002) Spotlight on the neurones (II): electrophysiology of the neurones in the perirhinal and entorhinal cortices and neuromodulatory changes in firing patterns. In: The parahippocampal region: organization and role in cognitive function (Witter MP, Wouterlood FG, eds), pp 89-105. Oxford: Oxford UP.

Bartesaghi R, Gessi T, Sperti L (1988) Electrophysiological analysis of the dorsal hippocampal commissure projections to the entorhinal area. Neuroscience 26:55-67.

Bartesaghi R, Di Maio V, Gessi T (2005) Topographic activation of the medial entorhinal cortex by presubicular commissural projections. J Comp Neurol 487:283-299.

Bartolomei F, Wendling F, Regis J, Gavaret M, Guye M, Chauvel P (2004) Pre-ictal synchronicity in limbic networks of mesial temporal lobe epilepsy. Epilepsy Res 61:89-104.

Bear J, Fountain NB, Lothman EW (1996) Responses of the superficial entorhinal cortex in vitro in slices from naive and chronically epileptic rats. J Neurophysiol 76:2928-2940.
Bragin A, Engel Jr J, Wilson CL, Fried I, Mathern GW (1999) Hippocampal and entorhinal cortex high-frequency oscillations $(100-500 \mathrm{~Hz})$ in human epileptic brain and in kainic acid-treated rats with chronic seizures. Epilepsia 40:127-137.

Bragin A, Mody I, Wilson CL, Engel Jr J (2002) Local generation of fast ripples in epileptic brain. J Neurosci 22:2012-2021.

Caballero-Bleda M, Witter MP (1993) Regional and laminar organization of projections from the presubiculum and parasubiculum to the entorhinal cortex: an anterograde tracing study in the rat. J Comp Neurol 328:115-129.

Caballero-Bleda M, Witter MP (1994) Projections from the presubiculum and the parasubiculum to morphologically characterized entorhinalhippocampal projection neurons in the rat. Exp Brain Res 101:93-108.

Cavazos JE, Golarai G, Sutula TP (1992) Septotemporal variation of the supragranular projection of the mossy fiber pathway in the dentate gyrus of normal and kindled rats. Hippocampus 2:363-372.

Colder BW, Wilson CL, Frysinger RC, Chao LC, Harper RM, Engel Jr J (1996) Neuronal synchrony in relation to burst discharge in epileptic human temporal lobes. J Neurophysiol 75:2496-2508.

D’Attellis CE, Isaacson SI, Sirne RO (1997) Detection of epileptic events in electroencephalograms using wavelet analysis. Ann Biomed Eng 25:286-293.

Dhillon A, Jones RS (2000) Laminar differences in recurrent excitatory transmission in the rat entorhinal cortex in vitro. Neuroscience 99:413-422.

Dickson CT, Alonso A (1997) Muscarinic induction of synchronous population activity in the entorhinal cortex. J Neurosci 17:6729-6744.

Dickson CT, Magistretti J, Shalinsky MH, Fransen E, Hasselmo ME, Alonso A (2000) Properties and role of $I_{\mathrm{h}}$ in the pacing of subthreshold oscillations in entorhinal cortex layer II neurons. J Neurophysiol 83:2562-2579.

Du F, Whetsell Jr WO, Abou-Khalil B, Blumenkopf B, Lothman EW, Schwarcz R (1993) Preferential neuronal loss in layer III of the entorhinal cortex in patients with temporal lobe epilepsy. Epilepsy Res 16:223-233.

Du F, Eid T, Lothman EW, Kohler C, Schwarcz R (1995) Preferential neuronal loss in layer III of the medial entorhinal cortex in rat models of temporal lobe epilepsy. J Neurosci 15:6301-6313.

Dugladze T, Heinemann U, Gloveli T (2001) Entorhinal cortex projection cells to the hippocampal formation in vitro. Brain Res 905:224-231.

Eid T, Jorritsma-Byham B, Schwarcz R, Witter MP (1996) Afferents to the seizure-sensitive neurons in layer III of the medial entorhinal area: a tracing study in the rat. Exp Brain Res 109:209-218.

Eid T, Schwarcz R, Ottersen OP (1999) Ultrastructure and immunocytochemical distribution of GABA in layer III of the rat medial entorhinal cortex following aminooxyacetic acid-induced seizures. Exp Brain Res 125:463-475.

Fountain NB, Bear J, Bertram III EH, Lothman EW (1998) Responses of deep entorhinal cortex are epileptiform in an electrogenic rat model of chronic temporal lobe epilepsy. J Neurophysiol 80:230-240.

Freeman JA, Nicholson C (1975) Experimental optimization of current source-density technique for anuran cerebellum. J Neurophysiol 38:369-382.

Funahashi M, Stewart M (1998) GABA receptor-mediated post-synaptic potentials in the retrohippocampal cortices: regional, laminar and cellular comparisons. Brain Res 787:19-33.

Germroth P, Schwerdtfeger WK, Buhl EH (1989) Morphology of identified entorhinal neurons projecting to the hippocampus. A light microscopical study combining retrograde tracing and intracellular injection. Neuroscience 30:683-691.

Gloveli T, Schmitz D, Heinemann U (1998) Interaction between superficial layers of the entorhinal cortex and the hippocampus in normal and epileptic temporal lobe. Epilepsy Res 32:183-193.

Gloveli T, Egorov AV, Schmitz D, Heinemann U, Muller W (1999) Carbachol-induced changes in excitability and $\left[\mathrm{Ca}^{2+}\right]_{\mathrm{i}}$ signalling in projection cells of medial entorhinal cortex layers II and III. Eur J Neurosci 11:3626-3636.

Gloveli T, Dugladze T, Schmitz D, Heinemann U (2001) Properties of entorhinal cortex deep layer neurons projecting to the rat dentate gyrus. Eur J Neurosci 13:413-420.

Haug FM (1976) Sulphide silver pattern and cytoarchitectonics of parahippocampal areas in the rat. Special reference to the subdivision of area 
entorhinalis (area 28) and its demarcation from the pyriform cortex. Adv Anat Embryol Cell Biol 52:3-73.

Heinemann U, Zhang CL, Eder C (1993) Entorhinal cortex-hippocampal interactions in normal and epileptic temporal lobe. Hippocampus 3:89-97.

Honda Y, Ishizuka N (2004) Organization of connectivity of the rat presubiculum: I. Efferent projections to the medial entorhinal cortex. J Comp Neurol 473:463-484.

Iijima T, Witter MP, Ichikawa M, Tominaga T, Kajiwara R, Matsumoto G (1996) Entorhinal-hippocampal interactions revealed by real-time imaging. Science 272:1176-1179.

Jones RS (1993) Entorhinal-hippocampal connections: a speculative view of their function. Trends Neurosci 16:58-64.

Jones RS (1994) Synaptic and intrinsic properties of neurons of origin of the perforant path in layer II of the rat entorhinal cortex in vitro. Hippocampus 4:335-353.

Jones RS, Heinemann U (1988) Synaptic and intrinsic responses of medical entorhinal cortical cells in normal and magnesium-free medium in vitro. J Neurophysiol 59:1476-1496.

Jones RS, Lambert JD (1990) Synchronous discharges in the rat entorhinal cortex in vitro: site of initiation and the role of excitatory amino acid receptors. Neuroscience 34:657-670.

Kloosterman F, Witter MP, Van Haeften T (2003a) Topographical and laminar organization of subicular projections to the parahippocampal region of the rat. J Comp Neurol 455:156-171.

Kloosterman F, Van Haeften T, Witter MP, Lopes Da Silva FH (2003b) Electrophysiological characterization of interlaminar entorhinal connections: an essential link for re-entrance in the hippocampal-entorhinal system. Eur J Neurosci 18:3037-3052.

Kobayashi M, Wen X, Buckmaster PS (2003) Reduced inhibition and increased output of layer II neurons in the medial entorhinal cortex in a model of temporal lobe epilepsy. J Neurosci 23:8471-8479.

Kohler C (1984) Morphological details of the projection from the presubiculum to the entorhinal area as shown with the novel PHA-L immunohistochemical tracing method in the rat. Neurosci Lett 45:285-290.

Kohler C (1985) Intrinsic projections of the retrohippocampal region in the rat brain. I. The subicular complex. J Comp Neurol 236:504-522.

Kohler C (1986) Intrinsic connections of the retrohippocampal region in the rat brain. II. The medial entorhinal area. J Comp Neurol 246:149-169.

Lopantsev V, Avoli M (1998) Laminar organization of epileptiform discharges in the rat entorhinal cortex in vitro. J Physiol (Lond) 509:785-796.

Lothman EW, Bertram III EH, Kapur J, Bekenstein JW (1996) Temporal lobe epilepsy: studies in a rat model showing dormancy of GABAergic inhibitory interneurons. Epilepsy Res Suppl 12:145-156.

Magistretti J, Ragsdale DS, Alonso A (1999) Direct demonstration of persistent $\mathrm{Na}^{+}$channel activity in dendritic processes of mammalian cortical neurones. J Physiol (Lond) 521:629-636.

Mathern GW, Babb TL, Leite JP, Pretorius K, Yeoman KM, Kuhlman PA (1996) The pathogenic and progressive features of chronic human hippocampal epilepsy. Epilepsy Res 26:151-161.

Mulders WH, West MJ, Slomianka L (1997) Neuron numbers in the presubiculum, parasubiculum, and entorhinal area of the rat. J Comp Neurol 385:83-94.

Pare D, deCurtis M, Llinas R (1992) Role of the hippocampal-entorhinal loop in temporal lobe epilepsy: extra- and intracellular study in the isolated guinea pig brain in vitro. J Neurosci 12:1867-1881.

Paxinos G, Watson C (1998) The rat brain in stereotaxic coordinates. San Diego: Academic.

Pitkanen A, Tuunanen J, Halonen T (1995) Subiculum, presubiculum and parasubiculum have different sensitivities to seizure-induced neuronal damage in the rat. Neurosci Lett 192:65-68.

Racine RJ (1972) Modification of seizure activity by electrical stimulation. II. Motor seizure. Electroencephalogr Clin Neurophysiol 32:281-294.

Sanna PP, Jirikowski GF, Lewandowski GA, Bloom FE (1992) Applications of DAPI cytochemistry to neurobiology. Biotech Histochem 67:346-350.

Scharfman HE (2002) The parahippocampal region in temporal lobe epilepsy. In: The parahippocampal region: organization and role in cognitive function (Witter MP, Wouterlood FG, eds), pp 321-340. Oxford: Oxford UP.

Scharfman HE, Goodman JH, Du F, Schwarcz R (1998) Chronic changes in synaptic responses of entorhinal and hippocampal neurons after aminooxyacetic acid (AOAA)-induced entorhinal cortical neuron loss. J Neurophysiol 80:3031-3046.

Sloviter RS (1982) A simplified Timm stain procedure compatible with formaldehyde fixation and routine paraffin embedding of rat brain. Brain Res Bull 8:771-774.

Spencer SS, Spencer DD (1994) Entorhinal-hippocampal interactions in medial temporal lobe epilepsy. Epilepsia 35:721-727.

Stewart M (1999) Columnar activity supports propagation of population bursts in slices of rat entorhinal cortex. Brain Res 830:274-284.

Tolner EA, van Vliet EA, Lopes da Silva FH, Witter MP, Gorter JA (2001) Presubicular projection to degenerated layer III of medial entorhinal area in a rat model for mesial temporal lobe epilepsy. Soc Neurosc Abstr 27:554.8.

Tolner EA, Van Vliet EA, Holtmaat AJ, Aronica E, Witter MP, Da Silva FH, Gorter JA (2003) GAP-43 mRNA and protein expression in the hippocampal and parahippocampal region during the course of epileptogenesis in rats. Eur J Neurosci 17:2369-2380.

Tolner EA, Gorter JA, Lopes da Silva FH, Heinemann U (2004) High frequent field activity and altered cellular responses in superficial layers of entorhinal cortex in brain slices from chronic epileptic rats. Soc Neurosci Abstr 30:228.16.

Tolner EA, Kloosterman F, Kalitzin SN, Lopes da Silva FH, Gorter JA (2005) Physiological changes in chronic epileptic rats are prominent in superficial layers of the medial entorhinal area. Epilepsia 46 [Suppl 5]:72-81.

van der Linden S, Panzica F, de Curtis M (1999) Carbachol induces fast oscillations in the medial but not in the lateral entorhinal cortex of the isolated guinea pig brain. J Neurophysiol 82:2441-2450.

Van Groen T, Lopes da Silva FH, Wadman WJ (1987) Synaptic organization of olfactory inputs and local circuits in the entorhinal cortex: a current source density analysis in the cat. Exp Brain Res 67:615-622.

van Haeften T, Jorritsma-Byham B, Witter MP (1995) Quantitative morphological analysis of subicular terminals in the rat entorhinal cortex. Hippocampus 5:452-459.

van Haeften T, Wouterlood FG, Jorritsma-Byham B, Witter MP (1997) GABAergic presubicular projections to the medial entorhinal cortex of the rat. J Neurosci 17:862-874.

van Haeften T, Baks-te-Bulte L, Goede PH, Wouterlood FG, Witter MP (2003) Morphological and numerical analysis of synaptic interactions between neurons in deep and superficial layers of the entorhinal cortex of the rat. Hippocampus 13:943-952.

van Vliet EA, Aronica E, Tolner EA, Lopes da Silva FH, Gorter JA (2004) Progression of temporal lobe epilepsy in the rat is associated with immunocytochemical changes in inhibitory interneurons in specific regions of the hippocampal formation. Exp Neurol 187:367-379.

Walther H, Lambert JD, Jones RS, Heinemann U, Hamon B (1986) Epileptiform activity in combined slices of the hippocampus, subiculum and entorhinal cortex during perfusion with low magnesium medium. Neurosci Lett 69:156-161.

Witter MP, Amaral DG (2004) The hippocampal region. In: The rat brain (Paxinos G, ed), pp 637-703. San Diego: Elsevier Academic.

Witter MP, Wouterlood FG, Naber PA, Van Haeften T (2000a) Anatomical organization of the parahippocampal-hippocampal network. Ann NY Acad Sci 911:1-24.

Witter MP, Naber PA, van Haeften T, Machielsen WC, Rombouts SA, Barkhof F, Scheltens P, Lopes da Silva FH (2000b) Cortico-hippocampal communication by way of parallel parahippocampal-subicular pathways. Hippocampus 10:398-410.

Woodhall GL, Bailey SJ, Thompson SE, Evans DI, Jones RS (2005) Fundamental differences in spontaneous synaptic inhibition between deep and superficial layers of the rat entorhinal cortex. Hippocampus 15:232-245.

Wouterlood FG (2002) Spotlight on the neurones (I): cell types, local connectivity, microcircuits, and distribution of markers. In: The parahippocampal region: organization and role in cognitive function (Witter MP, Wouterlood FG, eds), pp 61-88. Oxford: Oxford UP.

Wouterlood FG, Hartig W, Bruckner G, Witter MP (1995) Parvalbuminimmunoreactive neurons in the entorhinal cortex of the rat: localization, morphology, connectivity and ultrastructure. J Neurocytol 24:135-153.

Wouterlood FG, Van Haeften T, Eijkhoudt M, Baks-Te-Bulte L, Goede PH, Witter MP (2004) Input from the presubiculum to dendrites of layer-V neurons of the medial entorhinal cortex of the rat. Brain Res 1013:1-12. 